\title{
Dados de Carbono Molecular do Extrato Ciclo-hexano da Madeira de Eucalyptus urophylla S. T. Blacke por RMN de ${ }^{13} \mathrm{C}$
}

\author{
Natália Dias de Souza ${ }^{1}$, Heber dos Santos Abreu ${ }^{1}$, Tatiana de Felice Elias ${ }^{1}$, \\ João Vicente de Figueiredo Latorraca ${ }^{1}$, Jorge Mitiyo Maeda ${ }^{2}$
}

${ }^{1}$ Departamento de Produtos Florestais, Instituto de Florestas, Universidade Federal Rural do Rio de Janeiro - UFRRJ

${ }^{2}$ Departamento de Silvicultura, Instituto de Florestas, Universidade Federal Rural do Rio de Janeiro - UFRRJ

\section{RESUMO}

O conhecimento da composição química da madeira possibilita o entendimento de seu comportamento como matéria-prima para diversos usos, principalmente para a indústria de polpa celulósica, indústria de painéis, além de conferir propriedades preservativas face aos agentes bióticos e abióticos. Dentre as espécies do gênero Eucalyptus, destaca-se o Eucalyptus urophylla por apresentar o maior potencial de crescimento em termos de área plantada e em função da resistência ao fungo Cryphonectria cubensis, causador do cancro do eucalipto. O objetivo da pesquisa em tela foi analisar a possibilidade de propor um modelo sistemático de identificação para madeiras do gênero Eucalyptus por Ressonância Magnética Nuclear do ${ }^{13} \mathrm{C}\left(\mathrm{RMN}{ }^{13} \mathrm{C}\right)$. Neste trabalho, utilizaram-se os extratos ciclo-hexano de madeiras de clones de E. urophylla, devido a uma vasta diferença em deslocamento químico dos carbonos moleculares. O estudo está sendo aplicado também a outras espécies do gênero Eucalyptus. Os dados de $\mathrm{RMN}{ }^{13} \mathrm{C}$ mostraram que os espectros dos extratos ciclo-hexano do cerne foram os mais representativos para o estudo de clones de E. urophylla, devido ao maior número de absorções existentes. Com esses resultados, foi possível traçar o perfil espectral comum para a espécie E. urophylla de cada região (alburno, zona de transição e cerne) da madeira.

Palavras-chave: identificação, cerne, RMN.

\section{Molecular Carbon Data of Cyclohexane Extract from Eucalyptus urophylla S. T. Blacke Wood Through NMR ${ }^{13} \mathrm{C}$}

\begin{abstract}
The knowledge of the chemical composition of wood enables the understanding of its behavior as raw material for various uses, mainly in the pulp, wood panel and pharmacy industries, besides the fact that it confers preservative activity against biotic and abiotic agents. Within the Eucalyptus genus, the Eucalyptus urophylla presents the highest growth potentiality in relation to the planted area and in function of the resistance to Cryphonectria cubensis fungi, as promoters of eucalypts diseases. The objective of this research was to analyze the possibility to propose a systematic identification model for Eucalyptus genus wood through ${ }^{13} \mathrm{C}$ Nuclear Magnetic Resonance $\left(\mathrm{NMR}{ }^{13} \mathrm{C}\right)$. Cyclohexane extracts from wood clones of $E$. urophylla were used due to the differences in chemical displacement of the molecular carbons. The study is also being carried out with other species of the Eucalyptus genus. The results of the $\mathrm{NMR}{ }^{13} \mathrm{C}$ showed that the cyclohexane extracts spectra of heartwood were considered the most representative for the study in the E. urophylla clones, due to the largest number of existing spectrum signals. With these results, it was possible to identify a common spectral profile for each region of wood (sapwood, transition zone and heartwood) of E. urophylla species.
\end{abstract}

Keywords: identification, heartwood, NMR. 


\section{INTRODUÇÃO}

O gênero Eucalyptus pertence à família Myrtaceae, com aproximadamente 600 espécies, muitas variedades e híbridos. É na maioria representado por plantas de hábitos arbóreos, com alta taxa de crescimento e plasticidade (Oliveira et al., 1999).

Um fato mais recente relacionado ao gênero Eucalyptus é a existência de clones interligados ao melhoramento genético. A primeira plantação clonal foi estabelecida no Brasil em 1979, em uma área de 1000 ha no Estado do Espírito Santo, com ganhos expressivos em produtividade e qualidade da madeira (Ruy, 1998). A partir desse período a preferência pela clonagem tornou-se uma rotina na silvicultura do eucalipto, devido à consolidação dos ganhos a partir do melhoramento genético, promovendo a homogeneização das propriedades tecnológicas da madeira, com benefícios para a qualidade do produto final, altamente desejável na atividade industrial (Silva et al., 2003).

Dentre as espécies do gênero Eucalyptuscultivadas no Brasil, o E. urophylla é uma das espécies mais plantadas, ocupando uma área de aproximadamente seiscentos 1000 ha (Ferreira, 1999; Mora \& Garcia, 2000), já que apresenta o maior potencial de crescimento em termos de área plantada em função da resistência ao fungo Cryphonectria cubensis, causador do cancro do eucalipto (Ferreira, 1989). Tal resistência consegue ser superior à apresentada por procedências de E. grandis (Moura et al., 1980).

A primeira introdução de E. urophylla no Brasil foi realizada por Edmundo Navarro de Andrade antes de 1919 com o nome de E. alba. Em Rio Claro, SP, encontra-se o talhão mais antigo de E. urophylla, hoje com mais de 80 anos (Golfari et al., 1978).

A madeira de E. urophylla possui densidade mediana (Moura, 1986), cor clara com cerne de rosa a marrom. Tem sido utilizada para os mais diversos fins (produção de polpa celulósica e papel, chapas duras, serraria, produção de carvão entre outros). Essa diversidade de uso está correlacionada com a composição química da madeira, componentes estruturais e por diversas substâncias não pertencentes à parede celular (extrativos) (Sjöström \& Alén, 1998; Gullichsen \& Paulapuro, 2000).
Em muitos casos e principalmente o eucalipto à identificação botânica através de métodos usuais, muitas vezes esbarram em similaridades, (conflitos de observação), podendo chegar à melhor das hipóteses ao nível de gênero. $\mathrm{O}$ conhecimento do perfil químico das espécies vegetais é uma ferramenta interessante durante o processo de identificação e algumas classes de substâncias são utilizadas como marcadores quimiossistemáticos de um táxon botânico.

A utilização da técnica $\mathrm{RMN}{ }^{13} \mathrm{C}$ pode ser uma nova ferramenta para dirimir dúvidas na identificação de espécies botânicas lenhosas, já que esta permite elucidar as estruturas moleculares de várias substâncias que ocorrem em madeiras. Cienfuegos \& Vaitsman (2000) relatam as principais vantagens da RMN: preparação muito simples e fácil obtenção de dados de amostras das mais variadas formas e tamanhos.

$\mathrm{O}$ uso da $\mathrm{RMN}{ }^{13} \mathrm{C}$ aplicado rotineiramente para a verificação estrutural de substâncias da madeira, assim como as técnicas espectroscópicas avançadas $1 \mathrm{D}$ e $2 \mathrm{D}$, trouxeram avanços significativos para a elucidação estrutural de substâncias orgânicas encontradas em plantas. As técnicas DEPT, APT, HMQC, HMBC e HSQC, por exemplo, fornecem valores de correlação dos deslocamentos químicos dos carbonos e respectivos hidrogênios das substâncias (Harbone, 1994; Gottlieb \& Yoshida, 1989).

De uma forma geral, a RMN pode representar uma ferramenta interessante capaz de fornecer o perfil do carbono molecular para uma espécie, que dificilmente se repete integralmente em outras madeiras. Este trabalho teve como objetivo usar a técnica $\mathrm{RMN}{ }^{13} \mathrm{C}$ para propor um modelo sistemático de identificação utilizando madeiras oriundas de clones de E. urophylla por intermédio das variações espectrais apresentadas pelos seus extratos ciclohexano, considerando as variáveis sob o ponto de vista fisiológico, ecológico e genético.

\section{MATERIAL E MÉTODOS}

\subsection{Descrição botânica}

O E. urophylla é uma espécie nativa da Indonésia, do Arquipélago Sonda. Distribui-se principalmente 
entre $7^{\circ} 30^{\prime}$ a $10^{\circ}$ de latitude e 305 a 2960 m de altitude. A longitude varia de 122 a $127^{\circ}$ E. Nesta região, as precipitações anuais variam de 600 a $2500 \mathrm{~mm}$ e o clima é subtropical seco a tropical úmido com aproximadamente 4 a 5 meses secos por ano. A temperatura média máxima está entre $27-29^{\circ} \mathrm{C}$ aos $400 \mathrm{~m}$ de altitude e de 17 a $21^{\circ} \mathrm{C}$ a $1900 \mathrm{~m}$ de altitude (Martin \& Cossalter, 1975).

O E. urophylla possui duas variedades distintas, sendo uma de casca fibrosa, folhas lanceoladas, procedentes da ilha do Timor, de altitudes elevadas (acima de $1000 \mathrm{~m}$ ), variedade esta utilizada por Blacke (1977) para descrever a espécie e outra de casca lisa em diferentes proporções de fuste e folhas com formas e tamanhos variáveis (Martin \& Cossalter, 1975; Vieira \& Bucsan, 1980). Para Lorenzi et al. (2003), as árvores dessa espécie são perenifólias de 25-35 m de altura, com tronco ereto e cilíndrico, revestido por casca grossa dotada de fissuras finas longitudinais de cor cinza-escura. Ramagem oblíqua, formando copa alongada. Folhas simples, as jovens obovadas e as adultas oblanceoladas com base geralmente assimétrica, coriáceas, lustrosas, discolores (face inferior mais clara), com a nervura principal bem destacada, de margens inteiras e ápice longo-acuminado, de 10-19 m de comprimento, com pecíolo de 1,5-2,2 cm e de coloração clara.

\subsection{Coleta do material}

A coleta de material para análise foi realizada em parceria com a ARACRUZ FLORESTAL, S. A., localizada no Estado do Espírito Santo. As amostras foram provenientes de clones de árvores da espécie E. urophylla. Foram selecionadas 4 árvores da espécie em 2 áreas diferentes, dando preferência às adultas e saudáveis com idade aproximada de 15 anos
(Tabela 1). O material de estudo foi obtido em formato de disco com $5 \mathrm{~cm}$ de espessura e retirado do DAP (diâmetro à altura do peito).

\subsection{Preparação das amostras}

Em primeiro lugar, foram identificadas nos discos as regiões do alburno (porção de madeira que, em árvores vivas, contém células vivas e materiais de reserva (exemplo: amido); zona de transição (baixa permeabilidade; cor pálida ou branca; falta de amido, reduzido conteúdo de líquido e com largura entre 1-3 anéis de crescimento); e cerne (maior conteúdo de extrativos, baixa quantidade de água e células parenquimáticas mortas (Yang et al., 2004). De cada região retiraram-se amostras, das quais foram obtidos cavacos de madeira $(3 \mathrm{~cm}$ de comprimento e 0,6 $\mathrm{mm}$ de espessura), que foram convertidos em serragem em um moinho de facas do tipo Willey.

O material empregado nas análises foi aquele que passou pela peneira número 16 internacional (malha 40 ASTM) e o que ficou retido na peneira número 24 internacional (malha 60 ASTM).

\subsection{Obtenção dos extratos}

Empregou-se o aparelho de Soxhlet, utilizandose 16 g de madeira moída e homogênea de cada parte do disco de madeira. $\mathrm{O}$ material foi acondicionado em um cartucho confeccionado com papel filtro e colocado dentro do tubo de extração. O solvente foi colocado em um balão de $1000 \mathrm{~mL}$ obedecendo à escala eluitrópica na ordem crescente de polaridade (ciclo-hexano, acetado de etila e metanol). O tempo de extração para cada solvente foi de 48 horas ininterruptas. Logo após esse período, o balão contendo o material solúvel, foi concentrado em um rotavapor. Os concentrados foram transferidos para

Tabela 1. Material de clones de E. urophylla de duas áreas diferentes, coletado na Aracruz - ES.

Table 1. Material of E. urophylla clones from two different areas, collected in Aracruz - ES.

\begin{tabular}{|ccccccc} 
Área & Representação & Indivíduo & $\begin{array}{c}\text { Posição do } \\
\text { disco }\end{array}$ & $\begin{array}{c}\text { Altura da } \\
\text { árvore }\end{array}$ & $\begin{array}{c}\text { Material } \\
\text { genético }\end{array}$ & Espécie \\
\hline 162 & I & 1 & DAP & 38,50 & AR5 & E. urophylla \\
162 & I & 2 & DAP & 41,20 & AR5 & E. urophylla \\
406 & II & 1 & DAP & 44,90 & AR5 & E. urophylla \\
406 & II & 2 & DAP & 43,00 & AR5 & E. urophylla \\
\hline
\end{tabular}


um recipiente até a completa evaporação do solvente à temperatura ambiente.

\subsection{Análise estatística}

As análises estatísticas foram realizadas com o teste Kruskal-Wallis, considerando os efeitos da variação entre indivíduos e regiões da madeira estudada (alburno, zona de transição e cerne), bem como a interação entre os indivíduos versus solventes.

\section{6. $R M N N^{13} \mathrm{C}$}

A utilização de espectros de extratos brutos é limitada e nunca foi usada como ferramenta para caracterizar a madeira sob o ponto de vista da sistemática de identificação. Entretanto, a imensa quantidade de absorções que representam todos os carbonos moleculares pode significar uma impressão quase digital de uma determinada madeira. A relação dos deslocamentos químicos dos carbonos e o seu tipo podem trazer informações mais do que a existência de diferentes substâncias e pode ser um retrato da composição como um todo, cujas características refletem a natureza do lenho e sua origem genética.

Os espectros dos extratos foram obtidos em um espectrômetro VARIAN Mercury plus (300 $\mathrm{MHz}$ para ${ }^{1} \mathrm{H}, 75,46 \mathrm{MHz}$ para ${ }^{13} \mathrm{C}$ ), utilizando a sonda de $5 \mathrm{~mm}$ de diâmetro interno, à temperatura ambiente e pulso de $45^{\circ}$. Os deslocamentos químicos (ppm) foram referenciados no $\mathrm{RMN}^{13} \mathrm{C}$ ao $\mathrm{CDCl}_{3}$ (77 ppm) e $\mathrm{CD}_{3} \mathrm{OD}$ (49 ppm), respectivamente. O uso da técnica complementar APT (Attached Proton Test) com a sequência de pulsos auxiliou nas identificações e correlações dos carbonos (Breitmaier \& Voelter, 1987).

A obtenção dos espectros foi realizada no Centro de Ressonância Magnética Nuclear do Instituto de Química da Universidade de Brasília (UnB).

\section{RESULTADOS E DISCUSSÃO}

\subsection{Teores de extrativos}

Os teores dos extratos ciclo-hexano das madeiras das quatro árvores mantiveram-se quantitativamente constantes nas três regiões da madeira (alburno, zona de transição e cerne). Os teores dos extratos de acetato de etila apresentaram o mesmo comportamento. Quanto aos teores dos extratos metanólicos, diferenças foram observadas na zona de transição e no cerne (Tabela 2).

Nos alburnos, os teores dos extratos ciclo-hexano das madeiras das árvores 1 e 2 da área I e das árvores 1 e 2 da área II revelaram que não existem diferenças significativas, pressupondo que em todas as amostras o processo fisiológico manteve quantitativamente a mesma relação, significando que os clones estudados das regiões distintas não se modificaram, mantendo suas características genéticas quanto a esse fator.

Tabela 2. Teor de extrativos (\%) das três regiões (Alburno, Zona de transição e Cerne) da madeira de E. urophylla. Table 2. Extractives Content (\%) of the tree regions (sapwood, transition zone and heartwood) of E. urophylla wood.

\begin{tabular}{|c|c|c|c|c|c|c|c|c|c|}
\hline & \multicolumn{3}{|c|}{ Alburno } & \multicolumn{3}{|c|}{ Zona de transição } & \multicolumn{3}{|c|}{ Cerne } \\
\hline & $\begin{array}{l}\text { Ciclo- } \\
\text { hexano }\end{array}$ & $\begin{array}{l}\text { Acetato } \\
\text { de etila }\end{array}$ & Metanol & $\begin{array}{l}\text { Ciclo- } \\
\text { hexano }\end{array}$ & $\begin{array}{l}\text { Acetato } \\
\text { de etila }\end{array}$ & Metanol & $\begin{array}{l}\text { Ciclo- } \\
\text { hexano }\end{array}$ & $\begin{array}{l}\text { Acetato } \\
\text { de etila }\end{array}$ & Metanol \\
\hline $\begin{array}{l}\text { Indivíduo } 1 \text { - } \\
\text { área I }\end{array}$ & $0,12^{\mathrm{aA}}$ & $0,61^{\mathrm{aA}}$ & $2,37^{\mathrm{aA}}$ & $0,06^{\mathrm{aA}}$ & $0,66^{\mathrm{aA}}$ & $2,71^{\mathrm{aA}}$ & $0,18^{\mathrm{aA}}$ & $2,52^{\mathrm{aA}}$ & $8,43^{\mathrm{aB}}$ \\
\hline $\begin{array}{l}\text { Indivíduo } 2 \text { - } \\
\text { área I }\end{array}$ & $0,18^{\mathrm{aA}}$ & $0,43^{\mathrm{aA}}$ & $2,15^{\mathrm{aA}}$ & $0,06^{\mathrm{aA}}$ & $0,60^{\mathrm{aA}}$ & $4,39^{\mathrm{aB}}$ & $0,25^{\mathrm{aA}}$ & $1,11^{\mathrm{aA}}$ & $7,75^{\mathrm{aB}}$ \\
\hline $\begin{array}{l}\text { Indivíduo } 1 \text { - } \\
\text { área II }\end{array}$ & $0,23^{\mathrm{aA}}$ & $0,47^{\mathrm{aA}}$ & $3,58^{\mathrm{aA}}$ & $0,12^{\mathrm{aA}}$ & $0,12^{\mathrm{aA}}$ & $3,25^{\mathrm{aA}}$ & $0,30^{\mathrm{aA}}$ & $1,51^{\mathrm{aA}}$ & $7,01^{\mathrm{aB}}$ \\
\hline $\begin{array}{l}\text { Indivíduo } 2 \text { - } \\
\text { área II }\end{array}$ & $0,30^{\mathrm{aA}}$ & $0,30^{\mathrm{aA}}$ & $1,38^{\mathrm{aA}}$ & $0,24^{\mathrm{aA}}$ & $1,08^{\mathrm{aA}}$ & $7,87^{\mathrm{aB}}$ & $0,31^{\mathrm{aA}}$ & $0,98^{\mathrm{aA}}$ & $9,35^{\mathrm{aB}}$ \\
\hline
\end{tabular}

Valores seguidos da mesma letra (coluna/minúsculas) não diferem ao nível de 5\% de probabilidade, dentro de indivíduos/região pelo teste $\chi^{2}$. 
Quanto aos efeitos ecofisiológicos, por se tratarem de amostras de origem ecológicas diferentes, não sofreram modificações, revelando que no alburno não houve mudança significativa, o mesmo ocorreu para os teores dos extratos de acetato de etila. Já os teores dos extratos metanólicos da mesma região, os indivíduos 1 e 2 da área II destacaram-se dos demais, apresentando o maior e o menor teor, respectivamente, porém estatisticamente esta diferença não foi encontrada (Figura 1; Tabela 2).

Os teores dos extratos da madeira da zona de transição mostraram diferenças. Os extratos ciclo-hexano e acetato de etila foram semelhantes quantitativamente para todos os indivíduos. $\mathrm{O}$ mesmo não ocorreu para os extratos metanólicos, já que o indivíduo 2 da área II diferenciou-se dos demais. Estas diferenças não foram encontradas estatisticamente (Tabela 2). Isso mostra que os extratos polares foram mais influenciados com as diferentes condições ecológicas. Esse resultado pode ser explicado quando se trata a zona de transição como uma região com certa dinâmica de transformação bioquímica (Figura 2 e Tabela 2).

Os teores dos extratos da madeira do cerne mostraram-se diferenciados quantitativamente com relação aos extratos mais polares, confirmando que as substâncias polares neste caso foram as mais influenciadas pelo aspecto ambiental. Esta diferença não foi detectada estatisticamente (Figura 3; Tabela 2).

Essas variações podem ser explicadas enfocandose em três fatores: fisiológicos, ecológicos e genéticos.

A natureza e a quantidade de metabólitos especiais produzidos durante o desenvolvimento do vegetal podem ser afetadas pela radiação (alta ou baixa), temperatura (excessivamente elevada ou baixa), precipitação (alta, deficiente e seca total), ventos fortes, altitude, solo, época de coleta, entre outros (Evans, 1991; Vitti \& Brito, 1999; Larcher, 2000; Calixto, 2001; Gouinguené \& Turlings, 2002).

Neste caso, o comportamento fisiológico pode se manifestar muitas vezes dependendo de outros fatores. O estresse hídrico, as condições de nutrição e as condições edáficas, por exemplo, comprometem o funcionamento fisiológico normal, verificado nos indivíduos.

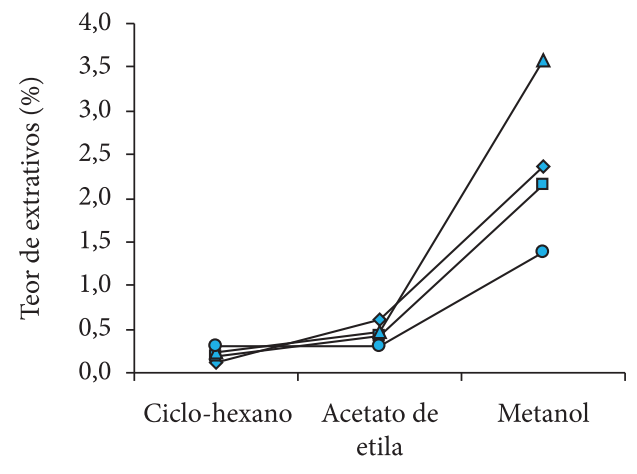

Alburno

$\multimap$ Indivíduo 1 área I $\square-$ Indivíduo 2 área I

$\triangle$ Indivíduo 1 área II $\multimap$ Indivíduo 2 área II

Figura 1. Valores em percentagens dos extratos ciclo-hexano , acetato de etila e metanol/peso seco da madeira, da região do alburno do lenho de E. urophylla dos indivíduos 1 e 2 das áreas I e II, respectivamente.

Figure 1. Values in percentage of extracts of cyclohexane, ethyl acetate and methanol/dry weight from the wood in the region of the log sapwood of E. urophylla from individuals, 1 and 2 of areas I and II, respectively.

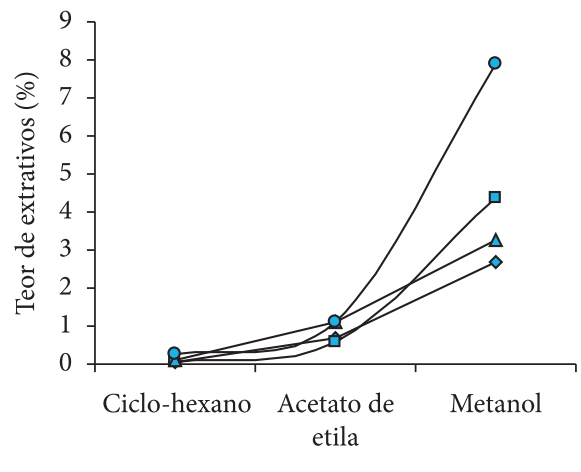

Zona de transição

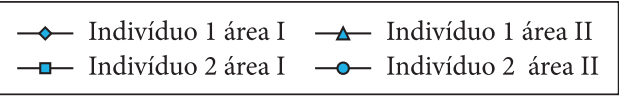

Figura 2. Valores em percentagens dos extratos ciclo-hexano , acetato de etila e metanol/peso seco da madeira, da região da zona de transição do lenho de E. urophylla dos indivíduos 1 e 2 das áreas I e II, respectivamente.

Figure 2. Values in percentage of extracts of cyclohexane, ethyl acetate and methanol/dry weight from the wood in the region of the log transition zone of E. urophylla from individuals, 1 and 2 of areas I and II, respectively. 
A ocorrência de déficit hídrico em plantas cultivadas afeta o crescimento e o desenvolvimento das culturas em todo o mundo (Santos \& Carlesso, 1998).

O déficit hídrico é uma situação comum à produção de muitas culturas, podendo apresentar um impacto negativo substancial no crescimento e desenvolvimento das plantas (Lecoeur \& Sinclair, 1996), assim, existe um conflito entre a conservação da água pela planta e a taxa de assimilação de $\mathrm{CO}_{2}$ para produção de carboidratos (Taiz \& Zeiger, 1991). A necessidade em se resolver este conflito leva a planta a desenvolver mecanismos morfofisiológicos, que as conduzem a economizar água para uso em períodos posteriores (McCree \& Fernández, 1989).

As respostas mais proeminentes das plantas ao déficit hídrico, segundo McCree \& Fernández (1989) e Taiz \& Zeiger (1991), consistem no decréscimo da produção da área foliar, no fechamento dos estômatos, na aceleração da senescência e na abscisão das folhas.

A demanda de nutrientes pela planta depende da sua taxa de crescimento e da eficiência com que ela converte os nutrientes absorvidos em biomassa. Para um mesmo material genético, numa determinada região, há uma relação relativamente estreita entre a taxa de crescimento e o acúmulo de nutrientes na biomassa (Barros et al., 2000). Entretanto, diferenças na eficiência nutricional entre procedências $e$ híbridos de eucalipto têm sido constatadas, podendo representar um fator importante na economia ou no emprego mais racional de fertilizantes (Molica, 1992; Paula et al., 1997).

As culturas florestais como qualquer população natural, estão sujeitas a uma série de fatores ecológicos que, direta ou indiretamente, podem afetar o crescimento das árvores, a produção de madeira, carvão e celulose. Algumas importantes contribuições são as perturbações causadas pela ação do fogo e da chuva. Gerando, muitas vezes, em espécies do gênero Eucalyptus um bolsão de material fenólico.

Fatores ambientais e genéticos, operando conjuntamente por meio de processos fisiológicos, controlam o crescimento e desenvolvimento das plantas. A luz, especialmente considerando-se a duração do período luminoso ou fotoperíodo, corresponde a um dos principais aspectos da interação das plantas com seu ambiente, controlando o seu desenvolvimento, a floração (Garner \& Allard, 1920), a germinação de sementes (Clemente Filha, 1996), o crescimento de caules e folhas (Juntilla et al., 1990), a formação de órgãos de reservas (Faria, 1998) e a partição de assimilados (Machácková et al., 1998).

Estes dados descritos na literatura levam-nos à reflexão sobre o uso de análise quantitativa, haja vista a complexidade dos fatores nos processos fisiológicos acumulativos diferenciados por diferentes condições de crescimento.

\section{2. $R M N{ }^{13} \mathrm{C}$}

Neste trabalho, foi avaliado o perfil espectral de $\mathrm{RMN}{ }^{13} \mathrm{C}$ dos extratos ciclo-hexano, visto que os espectros dos demais extratos (acetato de etila e metanólico) apresentaram-se pouco viáveis para identificação, dificultando assim à interpretação dos sinais

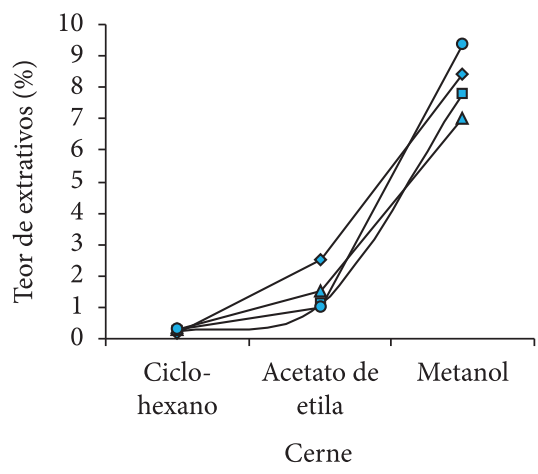

$\multimap$ Indivíduo 1 área I $\rightarrow$ Indivíduo 2 área I

$\triangle$ Indivíduo 1 área II $\multimap-$ Indivíduo 2 área II

Figura 3. Valores em percentagens dos extratos ciclo-hexano, acetato de etila e metanol/peso seco da madeira, da região do Cerne do lenho de E. urophylla dos indivíduos 1 e 2 das áreas I e II, respectivamente.

Figure 3. Values in percentage of dry extracts of cyclohexane, ethyl acetate and methanol/dry weight from the wood in the log region of the heartwood of E. urophylla from individuals, 1 and 2 of areas I and II, respectively. 
O extrato ciclo-hexano foi escolhido por vários aspectos: baixa complexidade do extrato, facilidade na obtenção dos espectros, maior solubilidade em clorofórmio Deuterado, tempo de obtenção menor em relação aos outros extratos polares. Outra característica deve-se ao fato que, quantitativamente, os extratos ciclo-hexano não apresentaram alterações nas condições ambientais e genéticas entre os indivíduos.
Os dados espectroscópicos de ${ }^{13} \mathrm{C}$ foram definidos segundo as regiões dos espectros: alifática (0 a 55 ppm), olefínica e aromática (110 a 155 ppm) e carbonílicas e acílicas (155 a 220 ppm).

As Tabelas 3, 4 e 5 apresentam o perfil espectral comum de cada região da madeira (alburno, zona de transição e cerne) para a espécie E. urophylla. Uma relação próxima entre os deslocamentos químicos dos extratos ciclo-hexano foi encontrada,

Tabela 3. Deslocamento químico em ppm dos extratos ciclo-hexano da região do alburno para as madeiras dos clones da espécie E. urophylla.

Table 3. Chemical displacement in ppm of cyclohexane extracts of the sapwood region from wood clones of E. urophylla species.

\begin{tabular}{|c|c|c|c|c|c|c|c|}
\hline \multicolumn{8}{|c|}{ Alburno } \\
\hline $\begin{array}{l}N^{\circ} \text { do } \\
\text { sinal }\end{array}$ & $\begin{array}{c}\text { Indivíduo } 1 \\
\text { área I }\end{array}$ & $\begin{array}{c}\text { Indivíduo } 2 \\
\text { área I }\end{array}$ & $\begin{array}{c}\text { Indivíduo } 1 \\
\text { área II }\end{array}$ & $\begin{array}{c}\text { Indivíduo } 2 \\
\text { área II }\end{array}$ & Média & Máxima & Mínima \\
\hline 1 & 11,83 & 11,83 & 11,83 & 11,83 & 8,87 & 11,83 & 11,83 \\
\hline 2 & 11,96 & 11,95 & 11,96 & 11,95 & 11,96 & 11,96 & 11,95 \\
\hline 3 & 14,12 & 14,04 & 14,09 & 14,11 & 14,09 & 14,12 & 14,04 \\
\hline 4 & 18,75 & 18,75 & 18,76 & 18,75 & 18,75 & 18,76 & 18,75 \\
\hline 5 & 19,00 & 19,00 & 19,02 & 19,00 & 19,00 & 19,02 & 19,00 \\
\hline 6 & 19,31 & 19,31 & 19,29 & 19,30 & 19,30 & 19,31 & 19,29 \\
\hline 7 & 19,81 & 19,80 & 19,79 & 19,80 & 19,80 & 19,81 & 19,79 \\
\hline 8 & 21,00 & 21,05 & 21,01 & 21,00 & 21,01 & 21,05 & 21,00 \\
\hline 9 & 22,68 & 22,68 & 22,67 & 22,67 & 22,67 & 22,68 & 22,67 \\
\hline 10 & 23,02 & 23,03 & 23,06 & 23,02 & 23,03 & 23,06 & 23,02 \\
\hline 11 & 23,70 & 23,71 & 23,74 & 23,70 & 23,71 & 23,74 & 23,70 \\
\hline 12 & 24,28 & 24,28 & 24,28 & 24,27 & 24,27 & 24,28 & 24,27 \\
\hline 13 & 25,03 & 25,02 & 25,03 & 25,02 & 25,02 & 25,03 & 25,02 \\
\hline 14 & 25,61 & 25,61 & 25,61 & 25,60 & 25,61 & 25,61 & 25,60 \\
\hline 15 & 25,99 & 26,02 & 26,08 & 26,02 & 26,03 & 26,08 & 25,99 \\
\hline 16 & 27,18 & 27,17 & 27,18 & 27,17 & 27,17 & 27,18 & 27,17 \\
\hline 17 & 27,78 & 27,79 & 27,80 & 27,78 & 27,78 & 27,80 & 27,78 \\
\hline 18 & 28,23 & 28,23 & 28,22 & 28,23 & 28,23 & 28,23 & 28,22 \\
\hline 19 & 28,89 & 28,90 & 28,91 & 28,90 & 28,90 & 28,91 & 28,89 \\
\hline 20 & 30,32 & 30,33 & 30,34 & 30,33 & 30,33 & 30,34 & 30,32 \\
\hline 21 & 34,70 & 34,69 & 34,69 & 34,69 & 34,69 & 34,70 & 34,69 \\
\hline 22 & 36,13 & 36,12 & 36,13 & 36,12 & 36,13 & 36,13 & 36,12 \\
\hline 23 & 36,56 & 36,57 & 36,59 & 36,57 & 36,57 & 36,59 & 36,56 \\
\hline 24 & 36,96 & 36,97 & 36,98 & 36,97 & 36,97 & 36,98 & 36,96 \\
\hline 25 & 37,21 & 37,22 & 37,24 & 37,22 & 37,22 & 37,24 & 37,21 \\
\hline 26 & 38,12 & 38,13 & 38,14 & 38,12 & 38,13 & 38,14 & 38,12 \\
\hline 27 & 68,13 & 68,14 & 68,15 & 68,14 & 68,14 & 68,15 & 68,13 \\
\hline 28 & 121,72 & 121,72 & 121,70 & 121,72 & 121,72 & 121,72 & 121,70 \\
\hline 29 & 122,58 & 122,59 & 122,56 & 122,58 & 122,58 & 122,59 & 122,56 \\
\hline 30 & 128,79 & 128,78 & 128,78 & 128,78 & 128,78 & 128,79 & 128,78 \\
\hline 31 & 130,90 & 130,87 & 130,85 & 130,87 & 130,87 & 130,90 & 130,85 \\
\hline
\end{tabular}


Tabela 4. Deslocamento químico em ppm dos extratos ciclo-hexano da região da zona de transição para as madeiras dos clones da espécie E. urophylla.

Table 4. Chemical displacement in ppm of cyclohexane extracts of the transition zone region from wood clones of E. urophylla species.

\begin{tabular}{rrrrrrrr}
\hline \multicolumn{7}{c}{ Zona de transição } \\
$\begin{array}{c}\text { No do } \\
\text { sinal }\end{array}$ & $\begin{array}{c}\text { Indivíduo 1 } \\
\text { área 1 }\end{array}$ & $\begin{array}{c}\text { Indivíduo 2 } \\
\text { área 1 }\end{array}$ & $\begin{array}{c}\text { Indivíduo 1 } \\
\text { área 2 }\end{array}$ & $\begin{array}{c}\text { Indivíduo 2 } \\
\text { área 2 }\end{array}$ & Média & Máxima & Mínima \\
\hline 1 & 11,96 & 11,96 & 11,95 & 11,94 & 11,95 & 11,96 & 11,94 \\
\hline 2 & 14,12 & 14,11 & 14,13 & 14,10 & 14,11 & 14,12 & 14,10 \\
\hline 3 & 18,75 & 18,75 & 18,74 & 18,74 & 18,75 & 18,75 & 18,74 \\
\hline 4 & 19,81 & 19,81 & 19,79 & 19,79 & 19,80 & 19,81 & 19,79 \\
\hline 5 & 22,68 & 22,68 & 22,67 & 22,66 & 22,67 & 22,68 & 22,66 \\
\hline 6 & 23,00 & 23,02 & 23,02 & 23,02 & 23,02 & 23,02 & 23,00 \\
\hline 7 & 23,70 & 23,07 & 23,70 & 23,70 & 23,70 & 23,70 & 23,70 \\
\hline 8 & 25,60 & 25,60 & 25,59 & 25,99 & 25,70 & 25,59 & 25,55 \\
\hline 9 & 27,17 & 27,18 & 27,17 & 27,18 & 27,17 & 27,18 & 27,17 \\
\hline 10 & 31,50 & 31,51 & 31,59 & 31,52 & 31,53 & 31,59 & 31,50 \\
\hline 11 & 33,89 & 33,90 & 33,90 & 33,89 & 33,89 & 33,90 & 33,89 \\
\hline 12 & 34,69 & 34,69 & 34,69 & 34,66 & 34,68 & 34,69 & 34,66 \\
\hline 13 & 36,12 & 36,13 & 36,12 & 36,12 & 36,12 & 36,13 & 36,12 \\
\hline 14 & 38,11 & 38,13 & 38,12 & 38,11 & 38,14 & 38,13 & 38,11 \\
\hline 15 & 38,67 & 38,69 & 38,69 & 38,67 & 38,68 & 38,69 & 38,67 \\
\hline 16 & 42,22 & 42,28 & 42,28 & 42,27 & 42,26 & 42,28 & 42,22 \\
\hline 17 & 45,78 & 45,79 & 45,79 & 45,77 & 45,78 & 45,79 & 45,77 \\
\hline 18 & 50,08 & 50,09 & 50,09 & 50,08 & 50,08 & 50,09 & 50,08 \\
\hline 19 & 68,14 & 68,13 & 68,14 & 68,13 & 68,13 & 68,14 & 68,13 \\
\hline 20 & 122,58 & 122,59 & 122,57 & 122,57 & 122,58 & 122,59 & 122,57 \\
\hline 21 & 127,87 & 127,89 & 127,88 & 127,88 & 127,88 & 127,89 & 127,87 \\
\hline 22 & 128,77 & 128,79 & 128,81 & 128,77 & 128,79 & 128,81 & 128,77 \\
\hline 23 & 130,88 & 130,88 & 130,90 & 130,87 & 130,88 & 130,90 & 130,87 \\
\hline
\end{tabular}

permitindo a obtenção de valores médios, ficando estes como os deslocamentos representativos para definir a espécie.

$\mathrm{O}$ alburno e o cerne foram as regiões que apresentaram as maiores faixas de deslocamentos químicos, enquanto a zona de transição, a menor. Esse resultado coaduna com a hipótese de que na zona de transição são formados metabólitos que se acumulam no cerne.

Os espectros dos extratos ciclo-hexano da região do alburno mostraram que os indivíduos 1 e 2 das áreas I e II apresentaram similaridade em torno de $\mathrm{n} \%$, revelando também que as absorções de ${ }^{13} \mathrm{C}$ (impressão digital do extrato ciclo-hexano) compõem alta concentração na região alifática $(\mathrm{C}$, $\mathrm{CH}, \mathrm{CH}_{2}$ e $\mathrm{CH}_{3}$ ), pouca concentração de carbonos olefínicos, e ausência de sinais de carbonos na região entre 80 e 110 ppm. As madeiras dos indivíduos estudados mantiveram, na maioria, as mesmas características espectrais, não ocorrendo alteração quanto à origem genética e ecológica (Tabela 3).

As absorções de ${ }^{13} \mathrm{C}$ nos espectros dos extratos ciclo hexano da zona de transição revelaram diferenças mínimas com relação ao alburno, com grande concentração de absorções na região alifática, embora em um número menor, confirmando o seu status de transição entre alburno e o cerne (Tabela 4). 
Tabela 5. Deslocamento químico em ppm dos extratos ciclo-hexano da região do cerne para as madeiras dos clones da espécie E. urophylla.

Table 5. Chemical displacement in ppm of extracts cyclohexane of the region of the heartwood from wood clones of E. urophylla species.

\begin{tabular}{|c|c|c|c|c|c|c|c|}
\hline \multicolumn{8}{|c|}{ Cerne } \\
\hline $\begin{array}{l}\text { No do } \\
\text { sinal }\end{array}$ & $\begin{array}{c}\text { Indivíduo } 1 \\
\text { área } 1\end{array}$ & $\begin{array}{c}\text { Indivíduo } 2 \\
\text { área } 1\end{array}$ & $\begin{array}{l}\text { Indivíduo } 1 \\
\text { área } 2\end{array}$ & $\begin{array}{c}\text { Indivíduo } 2 \\
\text { área } 2\end{array}$ & Média & Máxima & Mínima \\
\hline 1 & 11,83 & 11,83 & 11,82 & 11,80 & 11,82 & 11,83 & 11,80 \\
\hline 2 & 11,95 & 11,95 & 11,95 & 11,93 & 11,94 & 11,95 & 11,93 \\
\hline 3 & 14,09 & 14,11 & 14,10 & 14,08 & 14,10 & 14,11 & 14,08 \\
\hline 4 & 15,13 & 15,19 & 15,14 & 15,11 & 15,14 & 15,19 & 15,11 \\
\hline 5 & 18,75 & 18,75 & 18,74 & 18,72 & 18,74 & 18,75 & 18,72 \\
\hline 6 & 19,00 & 18,99 & 18,99 & 18,98 & 18,99 & 19,00 & 18,98 \\
\hline 7 & 19,79 & 19,80 & 19,80 & 19,78 & 19,79 & 19,80 & 19,78 \\
\hline 8 & 22,67 & 22,68 & 22,67 & 22,65 & 22,67 & 22,68 & 22,65 \\
\hline 9 & 23,03 & 23,02 & 23,00 & 22,99 & 23,01 & 23,03 & 22,99 \\
\hline 10 & 23,71 & 23,70 & 23,69 & 23,67 & 23,69 & 23,71 & 23,67 \\
\hline 11 & 24,28 & 24,28 & 24,26 & 24,25 & 24,26 & 24,28 & 24,25 \\
\hline 12 & 24,70 & 24,70 & 24,28 & 24,69 & 24,69 & 24,70 & 24,68 \\
\hline 13 & 26,03 & 26,00 & 25,98 & 25,97 & 25,99 & 26,03 & 25,97 \\
\hline 14 & 27,17 & 27,17 & 27,16 & 27,14 & 27,16 & 27,17 & 27,14 \\
\hline 15 & 27,98 & 28,08 & 27,93 & 27,93 & 27,98 & 28,08 & 27,93 \\
\hline 16 & 28,22 & 28,23 & 28,22 & 28,20 & 28,21 & 28,23 & 28,20 \\
\hline 17 & 28,75 & 28,74 & 28,73 & 28,72 & 28,73 & 28,75 & 28,72 \\
\hline 18 & 30,33 & 30,32 & 30,30 & 30,30 & 30,31 & 30,33 & 30,30 \\
\hline 19 & 31,90 & 31,90 & 31,89 & 31,87 & 31,89 & 31,90 & 31,87 \\
\hline 20 & 34,78 & 34,72 & 34,76 & 34,75 & 34,75 & 34,78 & 34,72 \\
\hline 21 & 36,12 & 36,12 & 36,11 & 36,09 & 36,11 & 36,12 & 36,09 \\
\hline 22 & 38,70 & 38,68 & 38,66 & 38,66 & 38,67 & 38,70 & 38,66 \\
\hline 23 & 39,74 & 39,68 & 39,71 & 39,66 & 39,70 & 39,74 & 39,66 \\
\hline 24 & 42,29 & 42,28 & 42,26 & 42,25 & 42,27 & 42,29 & 42,25 \\
\hline 25 & 45,80 & 45,78 & 45,77 & 45,76 & 45,78 & 45,80 & 45,76 \\
\hline 26 & 68,14 & 68,13 & 68,12 & 68,11 & 68,12 & 68,14 & 68,11 \\
\hline 27 & 109,27 & 109,25 & 109,23 & 109,27 & 109,24 & 109,27 & 109,23 \\
\hline 28 & 114,69 & 114,68 & 114,67 & 114,70 & 114,69 & 114,70 & 114,67 \\
\hline 29 & 123,02 & 123,03 & 123,02 & 123,00 & 123,02 & 123,03 & 123,00 \\
\hline 30 & 128,78 & 128,78 & 128,77 & 128,76 & 128,77 & 128,78 & 128,76 \\
\hline 31 & 130,03 & 130,05 & 130,03 & 130,00 & 130,03 & 130,05 & 130,00 \\
\hline 32 & 130,86 & 130,87 & 130,87 & 130,85 & 130,86 & 130,87 & 130,85 \\
\hline
\end{tabular}

Os espectros dos extratos ciclo-hexano provenientes da madeira da região do cerne foram considerados os mais representativos como parâmetro para o estudo de identificação de clones de E. urophylla, primeiro pelo número de absorções de ${ }^{13} \mathrm{C}$ existentes, significando uma variedade de carbonos em termos de constituintes químicos, além da presença de carbonos olefínicos e aromáticos entre 100 e 140 ppm (Tabela 5 e Figura 4).
Valores discrepantes de deslocamentos químicos foram encontrados entre os espectros dos extratos ciclo-hexano das madeiras das regiões (alburno, zona de transição e cerne). Essas diferenças podem estar relacionadas às baixas concentrações de alguns extratos, ao tempo de aquisição dos espectros, fatores genéticos e fatores ambientais. Por se tratar de um estudo com clones, indivíduos geneticamente idênticos, as diferenças observadas foram desprezadas. 

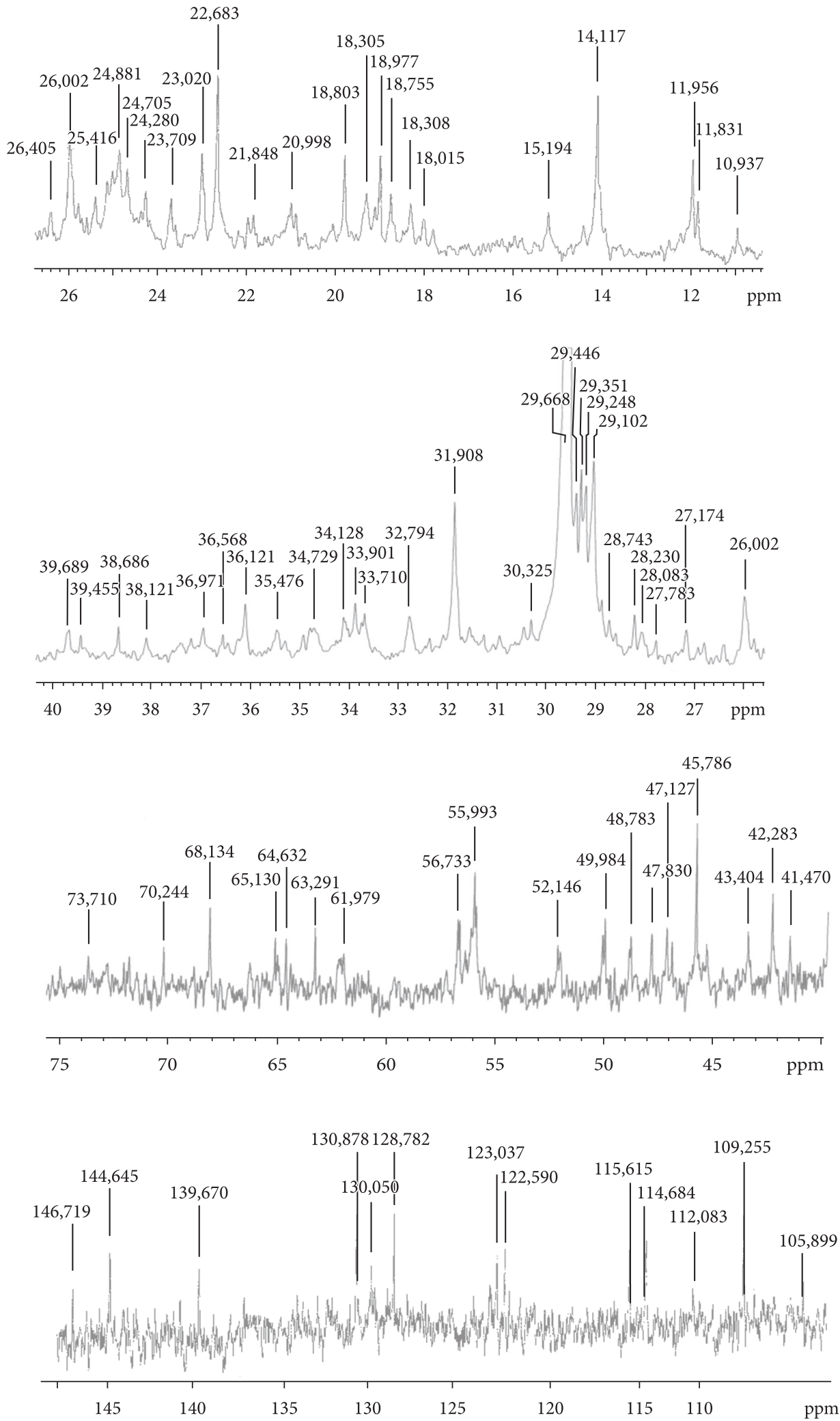

Figura 4. Espectros de $\mathrm{RMN}^{13} \mathrm{C}(\mathrm{CDCl} 3)$ do extrato ciclo-hexano da madeira do cerne de E. urophylla. Figure 4. NMR ${ }^{13} \mathrm{C}$ spectra of cyclohexane extract from heartwood of E. urophylla. 


\section{CONCLUSÕES}

Os dados espectroscópicos de $\mathrm{RMN} \quad{ }^{13} \mathrm{C}$ mostraram que os espectros dos extratos ciclohexano oriundos da madeira do cerne, foram considerados os mais representativos para o estudo de identificação de madeiras dos clones de E. urophylla, devido ao número de absorções existentes, quando comparados com os espectros dos extratos ciclo-hexano da madeira do alburno e da zona de transição. Neste sentido, foi possível identificar o perfil espectral comum dos extratos ciclo-hexano das madeiras de cada região (alburno, zona de transição e cerne) para espécie E. urophylla. Esses perfis espectrais deverão ser considerados de natureza específica para a espécie e funcionarão como dados importantes nas pesquisas de identificação da madeira por RMN ${ }^{13} \mathrm{C}$. Com esse estudo, novos perfis poderão ser traçados para diferentes espécies.

\section{AGRADECIMENTOS}

Os autores agradecem à CAPES o apoio financeiro através da concessão de bolsa do Programa de Pósgraduação em Ciências Ambientais e Florestais, à Aracruz Celulose S. A., em nome do Dr. Robert Sartório, a seção das amostras ao Instituto de Florestas da UFRRJ, ao Laboratório de Ressonância Magnética Nuclear da UnB os espectros de RMN ${ }^{13} \mathrm{C}$ e à FAPERJ o apoio financeiro para a aquisição de material.

\section{STATUS DA SUBMISSÃO}

Recebido: 22/03/2011

Aceito: 19/04/2011

Resumo publicado online: 11/05/2011

Artigo completo publicado: 30/06/2011

\section{AUTOR(ES) PARA CORRESPONDÊNCIA}

\section{Natália Dias de Souza}

Departamento de Produtos Florestais, Instituto de Florestas, Universidade Federal Rural do Rio de Janeiro - UFRRJ,

Rod. BR 465, Km 07, CEP 23890-000,

Seropédica, RJ, Brasil

e-mail: natdias@ufrrj.br

\section{REFERENNCIAS}

Barros NF, Neves JC, Novais RF. Recomendação de fertilizantes minerais em plantios de eucalipto. Piracicaba: IPEF; 2000. 427 p.

Blacke ST. Four New species of Eucalyptus. Austrobaileya 1977; 1(1):7-9.

Breitmaier E, Voelter W. Carbon-13 NMR Spectroscopy: high resolution methods and applications in organic chemistry and biochemistry. New York: VCH Publishers; 1987.400 p.

Calixto JB. Medicamentos Fitoterápicos. Santa Catarina: Editora Universitária UNOESC, Argos; 2001. 523 p.

Cienfuegos F, Vaitsman D. Análise instrumental. Rio de Janeiro: Interciência; 2000. 606 p.

Clemente Filha AC. Aspectos fisiológicos e fitoquímicos de Bauhinia forticata Link e Plantago major L [dissertação]. Lavras: Universidade Federal de Lavras, 1996.

Evans WC. Farmacognosia. México: Nueva Editorial Interamericana; 1991. 453p.

Faria LL. Influência do fotoperíodo no crescimento, composição química e indução de raízes tuberosas do feijão jacatupé (Pachyrrhizus tuberosus (Lam.) Spreng) [dissertação]. Lavras: Universidade Federal de Lavras, 1998 .

Ferreira F. Patologia Florestal - Principias Doenças Florestais no Brasil. Viçosa: Sociedade de Investigação Florestal; 1989. 570 p.

Ferreira M. Melhoramento genético do Eucalyptus urophylla S. T. Blake direcionado para a formação de populações base tipo casca lisa ("gum”). Piracicaba: FAPESP; 1999. $106 \mathrm{p}$.

Garner WW, Allard HA. Effect of relative lenght of day and night and others factors of the environment on growth and reproduction in plants. Journal of Agricultural Research 1920; 18(11):553-606.

Golfari L, Caser RL, Moura VPG. Zoneamento ecológico esquemático para reflorestamento no Brasil (2ªproximação). Brasília: PRODEPEF; 1978. 66 p.

Gottlieb OR, Yoshida M. Chemicals Extraneous to the lignocellulosic Cell Wall. Berlin: Springer Verlag; 1989. $1243 \mathrm{p}$

Gouinguené SP, Turlings TCJ. The effects of abiotic factors on induced volatile emissions in corn plants. Plant Physiology 2002; 129:1296-1307. PMid:12114583. PMCid:166523. http://dx.doi.org/10.1104/pp.001941

Gullichsen J, Paulapuro H. Forest products chemistry. Fapet Oy OyHelsinki; 2000. 350 p.

Harbone JB. The flavonoids advances in research since 1986. London: Chapman \& Hall; 1994. 611 p. 
Juntilla O, Svenning MM, Solheim B. Effects of temperature and photoperiod on frost resistance of white clover (Trifolium repens) ecotypes. Physiologia Plantarum 1990; 79(3):435-438. http://dx.doi. org/10.1111/j.1399-3054.1990.tb02099.x

Larcher W. Ecofisiologia vegetal. São Carlos: RiMa Artes e Textos; 2000. 531p.

Lecoeur J, Sinclair RT. Field pea transpiration and leaf growth in response to soil water deficits. Crop Science 1996; 36:331-335. http://dx.doi.org/10.2135/cropsci199 6.0011183X003600020020x

Lorenzi H, Souza HM, Torres MAV, Bacher LB. Árvores exóticas no Brasil: madeireiras, ornamentais e aromáticas. Nova Odessa, São Paulo: Instituto Plantarum; 2003. 382 p.

Machácková I, Konstantinova TN, Seergeva LI, Lozhnikova VN, Golyanovskaya SA, Dudko ND et al. Photoperiodic control of growth, development and phytohormone balance in Solanum tuberosum. Physiologia Plantarum 1998; 102(2):272-278. http:// dx.doi.org/10.1034/j.1399-3054.1998.1020215.x

Martin B, Cossalter C. Les Eucalyptus des lles de la Sonda. Bois et Forêts des Tropiques 1975; 163:3-25.

McCree KJ, Fernández CJ. Simulation model for studying physiological water stress responses of whole plants. Crop Science 1989; 29:353-360. http://dx.doi. org/10.2135/cropsci1989.0011183X002900020025x

Molica SG. Produção de biomassa e eficiência nutricional de híbridos interespecíficos de eucalipto em duas regiões bioclimáticas de Minas Gerais [tese]. Viçosa: Universidade Federal de Viçosa; 1992.

Mora AL, Garcia CH. A cultura do eucalipto no Brasil. São Paulo: SBS; 2000. 112 p.

Moura VPG. Provenance variation of Eucalyptus camaldulenses Den, in Brazil [tese]. Oxford: Oxford University; 1986. 304 p.

Moura VPG, Caser RL, Albino JC, Guimarães DP, Melo JT, Comastri SA. Avaliação de espécies e procedências de Eucalyptus em Minas Gerais e Espírito Santo: resultados parciais. Planaltina: EMBRAPA-CPAC; 1980. 140 p. (Boletim de Pesquisa 1).

Oliveira JTS, Hellmeister JC, Simões JW, Tomazello Filho, M. Caracterização da madeira de sete espécies de eucaliptos para a construção civil: 1- avaliações dendrométricas das árvores. Scientia Forestalis 1999; 56:113-124.

Paula RC, Pires IE, Paula NF, Barros NF, Borges RCG. Exportação de nutrientes por famílias de meio-irmãos de Eucalyptus camaldulensis Dehnh. In: Proceedings of the IUFRO Conference on Silviculture and Improvement of Eucalypts; 1997; Colombo. Colombo: Embrapa CNPF;1997. v. 1, p. 200-205.

Ruy OF. Variação da qualidade da madeira em clones de Eucalyptus urophylla S. T. Blake da Ilha de Flores, Indonésia [dissertação]. Piracicaba: Universidade de São Paulo; 1998.

Santos RF, Carlesso R. Déficit hídrico e os processos morfológicos e fisiológicos das plantas. Revista Brasileira de Engenharia Agrícola e Ambiental 1998; 2(3):287-294.

Silva JC, Asis TF, Oliveira JT. Melhoramento genético para a qualidade da madeira. Revista da Madeira 2003; $75: 10$.

Sjöström E, Alén R. Analytical methods in wood chemistry, pulping, and papermaking. Berlin: SpringerVerlag; 1998. 316 p.

Taiz L, Zeiger E. Plant Physiology. Redwood City, California: The Benjamim/ Cummings Publishing Company, Inc.; 1991.559 p.

Vieira FS, Bucsan B. Ocorrências naturais de E. urophylla na Indonésia. Silvicultura 1980; 14:359-361.

Vitti AM, Brito JO. Produção de óleo essencial de eucalipto. IPEF Noticias 1999; 23(146):11.

Yang J, Kamdem DP, Keaathley DE, Hwan Han K. Seasonal changes in gene expression at the sapwoodheartwood transition zone of black locust (Robinia pseudoacacia) revealed by cDNA microarray analysis. Tree Physiology 2004; 24:461-474. 